\title{
Influence of interleukin-8 (IL-8) and IL-8 receptors on the migration of human keratinocytes, the role of PLC- $\gamma$ and potential clinical implications
}

\author{
WEN G. JIANG, ANDREW J. SANDERS, FIONA RUGE and KEITH G. HARDING
}

Departments of Surgery and Wound Healing, Cardiff University School of Medicine, Heath Park, Cardiff CF14 4XN, UK

Received September 19, 2011; Accepted November 2, 2011

DOI: $10.3892 / \mathrm{etm} .2011 .402$

\begin{abstract}
Interleukin (IL)-8 is a pro-inflammatory cytokine that has a direct effect on immune cells, including polymorphonuclear cells. Keratinocytes are a rich source of IL-8. However, there is little knowledge on the role of IL-8 in clinical wound healing and the direct biological effect of IL-8 on keratinocytes. In this study, the effect of recombinant human IL-8 (rhIL-8) on migration and adhesion was tested using $\mathrm{HaCaT}$ keratinocytes as a cell model. The cell functions were evaluated using impedance cell sensing. The expression of IL-8 receptor (IL-8R) transcripts in human skin and wounds (acute and chronic) was assessed using real-time transcript analysis. rhIL-8 significantly increased the migration of keratinocytes (3.5 \pm 0.3 for cells treated with IL-8 vs. 2.7 \pm 0.6 for controls; $\mathrm{p}=0.029$ ). It is interesting to note that treatment of keratinocytes with IL-8 resulted in a marked shift in the responsive frequencies. IL-8 only resulted in a marginal increase in cell adhesion, which was particularly noticeable at high frequencies. The PLC- $\gamma$ inhibitor completely eradicated the action of IL-8 on the migration of HaCaT cells. Using real time PCR, it was found that chronic wounds had significantly lower levels of the $B$ form of the IL-8R (IL-8RB) $(p=0.045)$ and marginally lower levels of the A form, IL-8RA, in comparison with acute wounds. Therefore, IL-8 has a direct and profound stimulatory effect on the migration of human keratinocytes, which is likely to occur via the PLC- $\gamma$ pathway. Together with a reduced level of IL-8Rs in difficult-healing wounds, IL-8 has a clear prognostic and therapeutic value in wound healing.
\end{abstract}

\section{Introduction}

Interleukin (IL)-8, also known as neutrophil activating peptide, CXCL8, small inducible cytokine subfamily B (SCY88) and granulocyte chemotactic protein-1 (GCP-1), is a cytokine that

Correspondence to: Professor Wen G. Jiang, Metastasis and Angiogenesis Research Group, Cardiff University School of Medicine, Heath Park, Cardiff CF14 4XN, UK

E-mail: jiangw@cf.ac.uk

Key words: wound healing, interleukin-8, interleukin-8 receptor, PLC- $\gamma$, keratinocytes, skin, tissue repair, cellular migration belongs to the CXC chemokine family $(1,2)$. IL-8 is a small protein that is encoded by the $I L-8$ gene, which is located on chromosome 4q12-q21 (3). IL-8 has been shown to be linked to a number of conditions including viral bronchiolitis (4-6), cystic fibrosis related pathophysiology of the lungs, infection and other inflammatory diseases, including rheumatoid arthritis (7). It plays a pivotal role in the pathophysiology of sepsis. IL-8 is a powerful chemoattractant for neutrophils, basophils and T cells, but not monocytes, although the latter is a rich source of IL-8.

The action of IL-8 is mediated by its receptor, IL-8R. IL-8R has two forms, IL-8RA and IL-8RB. IL-8RA is also known as chemokine, CXC motif, receptor-1 (CXCR1), or IL-8R1. $I L-8 R A$ is located on chromosome $2 \mathrm{q} 35(8,10)$. IL-8RB is also known as chemokine, CXC motif, receptor-2 (CXCR2) or IL-8R2 $(8,9)$, and is located on chromosome $2 \mathrm{q} 35$. In the body, monocytes and fibroblasts are rich sources of IL-8. It was recently reported that the healing of diabetic and non-diabetic leg ulcers following treatment with Lactobacillus plantarum, increased such that, to a certain degree, it coincided with the increase in the IL- 8 production of the polymorphonuclear cells in the wound base (11). It has also been shown that IL-8 has an impact on the migration of fibroblasts and endothelial cells.

IL- 8 has been found to be highly stained in keratinocytes (12). Keratinocytes increased the expression of IL-8 in response to inflammatory stimuli such as tissue plasminogen activator (tPA) (13). Other agents, include trypsin, which increases and salbutamol, which inhibits the production of IL-8 from keratinocytes (14). Gingko biloba extract also inhibits IL-8 secretion from keratinocytes (15). IL-8RA and IL-8RB have been found to be present in keratinocytes of the skin. IL-8 and IL-8R have also been shown to be co-expressed in gingival keratinocytes (16). In psoriasis, IL-8R has been found to be overexpressed in keratinocytes, which may be inhibited by FK-506, an anti-psoriasis compound (17).

The potential role of IL-8 as a cell migration and chemotaxis factor indicates a significant role in wound healing. Indeed, it has been demonstrated that IL-8 may be chemotactic for human keratinocytes, which express IL-8Rs (18). In animal models using IL-8RB knockout mice, there was a delayed rate of wound healing (19). This appears to be associated with a delay in monocyte recruitment to the wounding space and a delay in re-epithelialisation and angiogenesis.

The impact of IL- 8 and the IL- $8 \mathrm{R}$ in the context of chronic wounds and clinical wound healing have otherwise not been 
well investigated. Chronic wounds represent a major health issue. Derived from multiple aetiology, including diabetes mellitus, pressure sores and circulation conditions, chronic wounds are costly to treat for the healthcare system. For example, in the UK, there are approximately 200,000 patients with chronic wounds at any given time, the care of which consumes more than $3 \%$ of the budget of the NHS. However, the biology of wound healing and, in particular, chronic wound healing are not yet fully understood. Wound healing is a complex biological process that requires temporal, special and co-ordinated action of multiple cell types, including immune cells for combating possible pathogens, fibroblasts for matrix deposition and remodelling, endothelial cells for the angiogenic process and keratinocytes for re-epithelialisation. IL-8 appears to play multiple roles in these processes.

In the present study, we investigated the transcript expression of IL-8RA and IL-8RB in a cohort of human wound tissues, and report the significant link between the cytokine complex and the fate of the wound. Furthermore, we have shown that IL-8 has a direct effect on the migration of human keratinocytes.

\section{Materials and methods}

Cells and tissues. The human keratinocyte cell line, HaCaT, was purchased from the German Cancer Institute and routinely maintained in Dulbecco's Modified Eagle's Medium (DMEM), supplemented with $10 \%$ fetal calf serum (FCS) and antibiotics. A cohort of fresh, frozen human wound tissues, acute wound tissues $(n=10)$ and chronic wound tissues from venous ulcers $(n=14)$, were collected under an Ethics Approval from clinics in the University Hospital of Wales, and used for histology and gene expression analysis, as previously reported $(20,21)$.

Recombinant human IL-8 (rhIL-8) was obtained from NBSB (Potters Bar, England, UK). Antibodies to human IL-8RA and IL-8RB were from Santa Cruz Biotechnologies Inc. (Santa Cruz, CA, USA). ERK inhibitor II (FR180204) and PLC- $\gamma$ inhibitor (U73122) were purchased from Calbiochem (Merck Chemicals Ltd, Nottingham, UK). FAK inhibitor was from Tocris (Bristol, England), and Arp2/3 inhibitor was from Chemidiv Ltd (San Diego, CA, USA).

Tissue processing and preparation of materials for protein and molecular investigations. Tissues were frozen and sectioned on a Leica cryostat at 5-10 $\mu \mathrm{M}$ thickness. The sections were divided into two portions: one section was processed for histological investigation and the other for extraction of genetic materials. For histological analysis, sections were laid on slides, prior to fixing and blocking by hydrogen peroxide and methanol. The section was then processed for H\&E staining.

A large number of frozen sections were combined and homogenised using a homogenizer in a RNA isolation buffer (Triagent; Sigma-Aldrich, Poole, Dorset, UK). Total RNA was subsequently separated and quantified using a spectrophotometer.

Quantitative analysis of $I L-8 R A$ and $I L-8 R B$ transcripts in cells and tissues. Total RNA was extracted from cell pellets using Triagent obtained from Sigma-Aldrich. Fresh frozen wound tissues were combined, resuspended in Triagent and homogenised using a hand-held homogeniser (Fisher Scientific). Total RNA was extracted and purified from the cells and tissue sections according to manufacturer's instructions. Equal amounts of RNA were reverse transcribed into complementary DNA (cDNA) using a first-strand reverse transcription (RT) kit from Bio-Rad (Hemel Hemstead, UK). For quantitative analysis of the IL-8R transcripts, we employed a real-time quantitative PCR assay, as previously reported (22-24). Briefly, primers (sequences shown in Table I) were designed using the Beacon Designer software and satisfied the following criteria: the amplified region sits on an exon-exon joint region and is specific to the respective target; the amplicon is smaller than $150 \mathrm{bp}$; a sequence that was complementary to the probe was added to one of the primers (routinely the reverse primer); and the annealing temperature is, or is close to being, $55^{\circ} \mathrm{C}$. The Ampliflor Uniprimer system was used as the probe system. cDNA from cells and tissues, together with a set of standards were amplified simultaneously, on a Icycler ${ }^{\mathrm{IQ} 5}$ system (Bio-Rad). The concentration of the respective transcript was calculated from the standard curve, which was simultaneously generated. The levels of the transcripts are shown here as the respective transcript/glyceraldehyde 3-phosphate dehydrogenase (GAPDH) ratio.

Electric cell-substrate impedance sensing (ECIS)-based cell adhesion and cell migration assays. ECIS Ztheta and 96W1E arrays were used (Applied Biophysics Inc, Troy, NY, USA) $(23,25,26)$. Following treatment of the array surface with a cysteine solution, the arrays were incubated with complete medium for $1 \mathrm{~h}$. The same number of $\mathrm{HaCaT}$ cells were added into each well, with or without IL-8 and small inhibitors. Cell adhesion was recorded immediately following addition of the cells, at multiple frequencies, for up to $6 \mathrm{~h}$. For the cell migration assay, confluent $\mathrm{HaCaT}$ cells with or without IL-8, and small signalling inhibitors, were wounded for $20 \mathrm{sec}$ at $6 \mathrm{~V}$. The migration of the cells was immediately traced following wounding for up to $18 \mathrm{~h}$, again with multiple frequencies. Cell behaviour was modelled using the Rb method, using a cell-free well as a reference unit. Cell migration and adhesion are shown here as the change in resistance.

Statistical analysis was carried out using Minitab. For normality test, the Anderson-Darling test was used, and for statistical differences the Student's t-test and MannWhitney U test were used as appropriate.

\section{Results}

IL-8 markedly increased the migration of keratinocytes. Using electrical wounding methods, we first tested the impact of rhIL-8 on the migration of HaCaT cells following wounding. As seen in Fig. 1, IL-8 markedly increased the migration of the cells (Fig. 1).

Using 3-D cellular modelling, the differences between control and IL-8-treated cells were clearly visible (Fig. 2) with the maximum effects observed at a frequency of $4,000 \mathrm{~Hz}$. This difference was statistically significant (p<0.01) (Fig. 2).

IL-8 had a significant effect on the adhesion of keratinocytes. We further tested to see whether IL- 8 had an effect on the adhesion of keratinocytes to the matrix. Again, using the ECIS attachment method, we evaluated the rate of cell adhesion. As shown in Fig. 3, the presence of IL-8 markedly increased the adhesion of the cells. 
Table I. Primers used in the quantitative analysis of gene transcripts.

\begin{tabular}{lll}
\hline Molecule name & \multicolumn{1}{c}{ Forward primer $\left(5^{\prime}-3^{\prime}\right)$} & \multicolumn{1}{c}{ Reverse primer $\left(5^{\prime}-3^{\prime}\right)$} \\
\hline IL-8RA & TGGGGACTGTCTATGAATCT & ACTGAACCTGACCGTACACATTTCCCAGGACCTCATA \\
IL-8RB & TCAAATTCATATGTCTCAGCA & ACTGAACCTGACCGTACAGTTGCCCATGTCCTCATA \\
IL-8 & TCTCTTGGCAGCCTTCCT & ACTGAACCTGACCGTACATGTCTTTATGCACTGACATCT \\
GAPDH & CTGAGTACGTCGTGGAGTC & ACTGAACCTGACCGTACACAGAGATGATGACCCTTTTG
\end{tabular}

GAPDH, glyceraldehyde 3-phosphate dehydrogenase; IL-8R, IL-8 receptor; IL, interleukin.
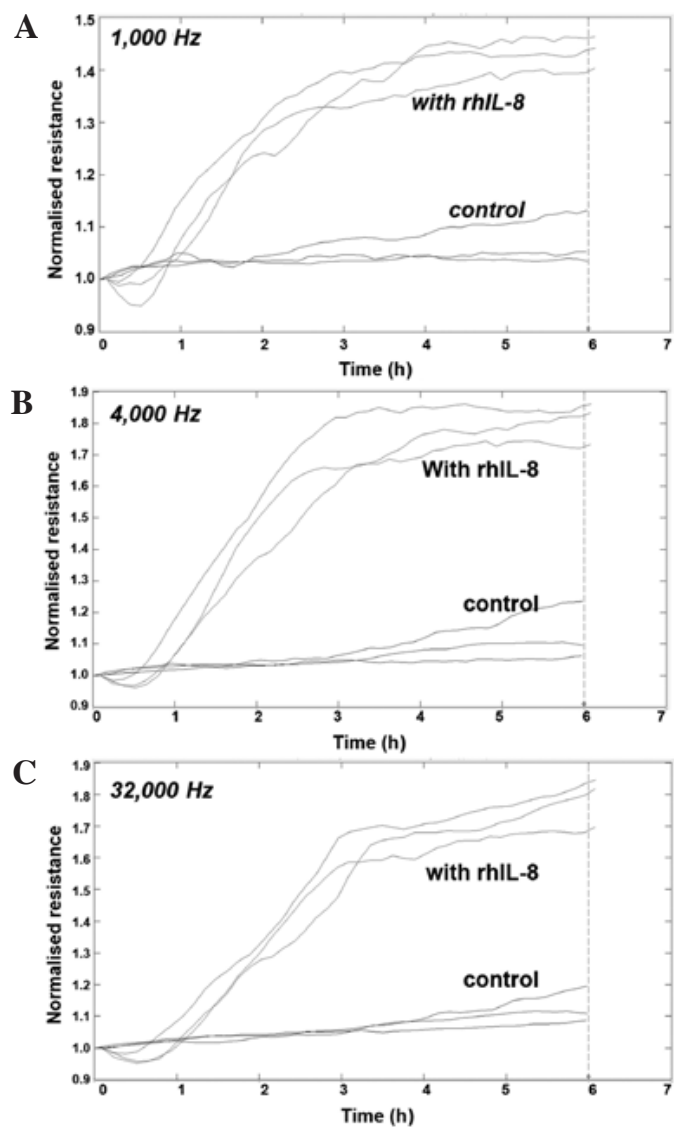

Figure 1. The effect of rhIL-8 on the migration of HaCaT cells. Confluent $\mathrm{HaCaT}$ cells in the 96W1E array were wounded for $20 \mathrm{sec}$ at $6 \mathrm{~V}$, following which the migration was immediately traced for up to $8 \mathrm{~h}$ over 12 frequencies [shown are (A) 1000, (B) 4000 and (C) $32000 \mathrm{~Hz}$ ]. rhIL-8 markedly increased the pace of migration of the cells. rhIL-8, recombinant human IL-8.

Potential signalling pathways in the action of $I L-8$. In order to explore potential pathways involved in the aforementioned action, we used a panel of small inhibitors to some of the known pathways downstream of the IL-8R. We have demonstrated that the PLC- $\gamma$ inhibitor markedly inhibited the increased migration induced by IL-8, and brought the migration to the control level. The same inhibitor has an inhibitory effect on cell adhesion, although this is yet to reach significance (data not shown). The ERK inhibitor also had an inhibitory effect on the migration of keratinocytes, but did not completely eliminate the action of IL-8. The FAK and ARP2/3 inhibitors had marginal effects (Fig. 4).
A

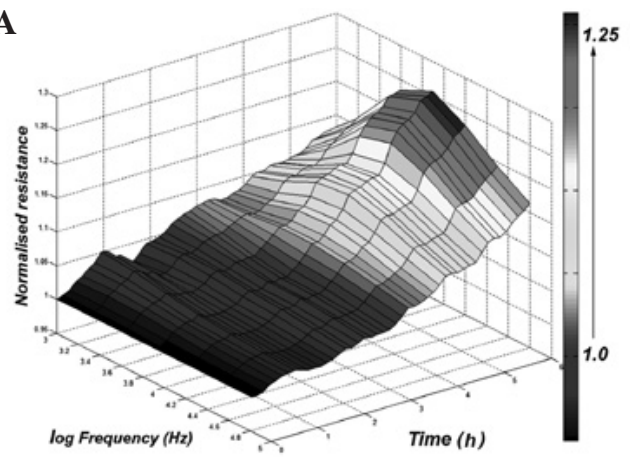

B
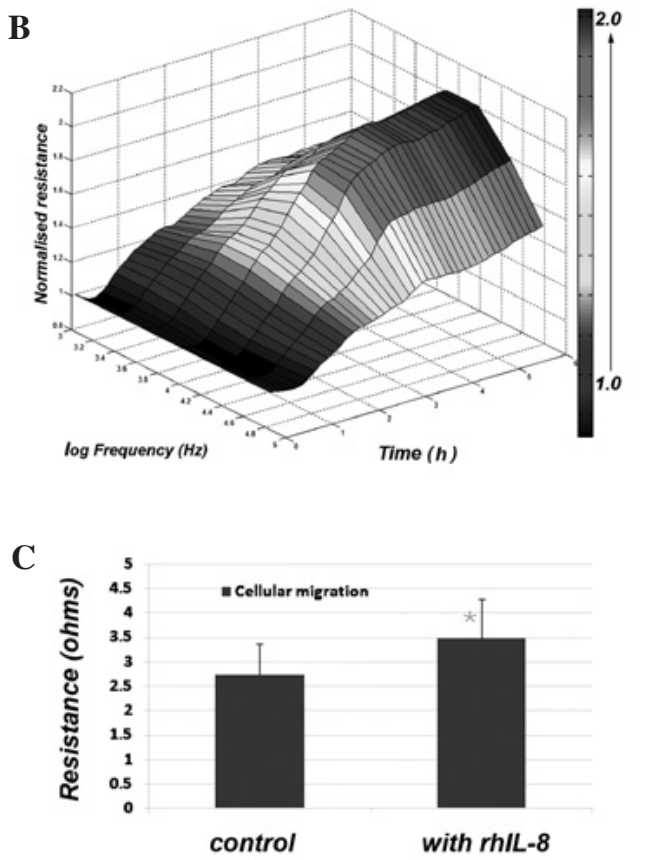

Figure 2. Effect of rhIL-8 on cellular migration of HaCaT cells, a 3-D demonstration. Cells were wounded in a similar fashion as in Fig. 1 and recorded following wounding over $7 \mathrm{~h}$. When treated with IL-8, cells migrated earlier (B) and faster than the control cells (B). (A and B) mean of 8 traces, (C) Cell modelling. " $\mathrm{p}<0.01$ vs. control cells. rhIL-8, recombinant human IL-8.

Human chronic wound tissues had a marked low level of $I L-8 R B$. We investigated the transcript levels of the IL-8Rs, IL-8RA and IL-8RB in acute and chronic wound tissues. As shown in Fig. 5, there were significantly lower levels of IL-8RB in chronic wound tissues than in acute tissues (Fig. 5). Although the levels of IL-8RA tended to be lower, this was not statistically significant. 
A

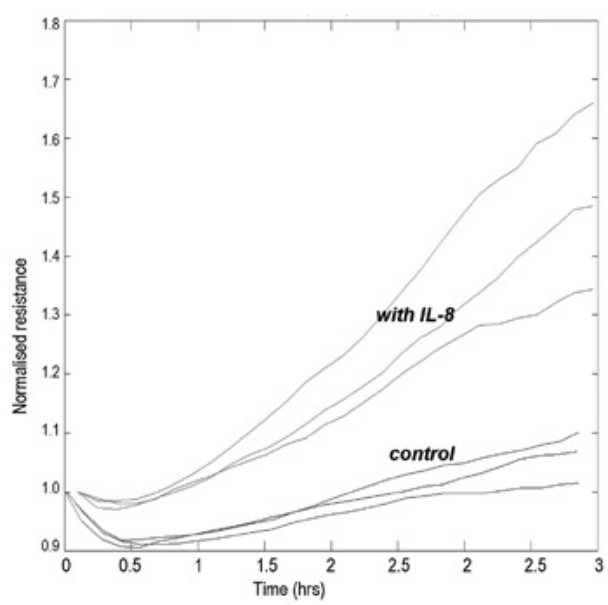

C

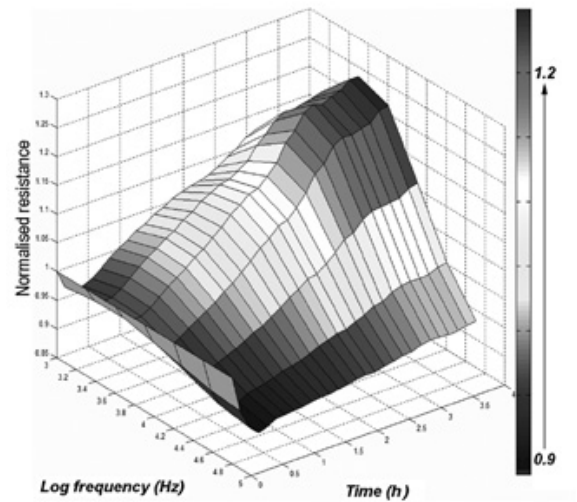

B

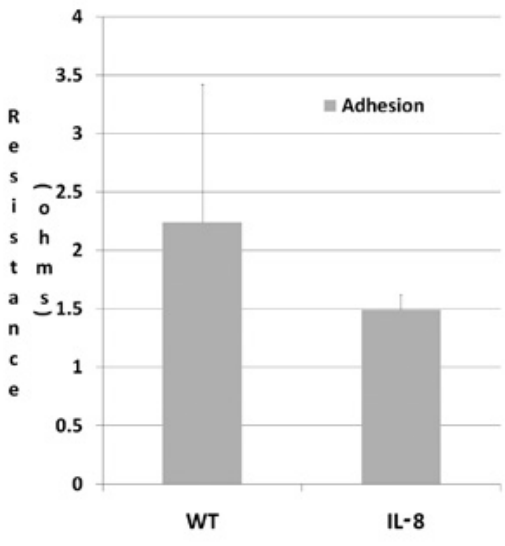

D

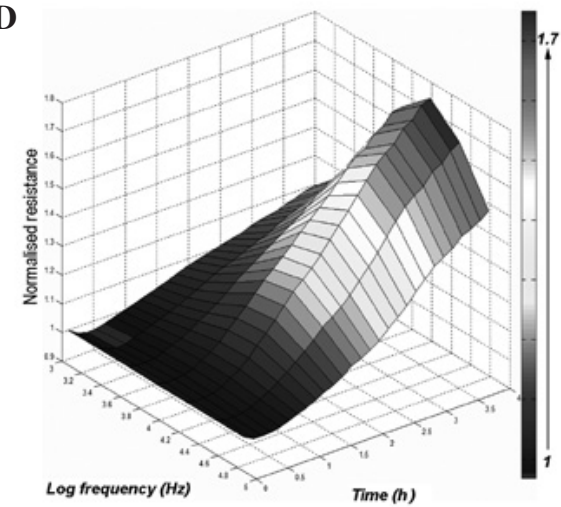

Figure 3. rhIL-8 had a significant impact on the adhesion of keratinocytes, assessed using the ECIS method. Cells were added to the ECIS arrays, pre-treated with $10 \%$ FCS; the adhesion was traced immediately following addition of the cells. (A) Traces of adhesion, (B) normalised resistance of adhesion traces, (C and D) 3-D modelling of adhesion (as resistance). rhIL-8, recombinant human IL-8; ECIS, electric cell-substrate impedance sensing; FCS, fetal calf serum; WT, wild-type.

A
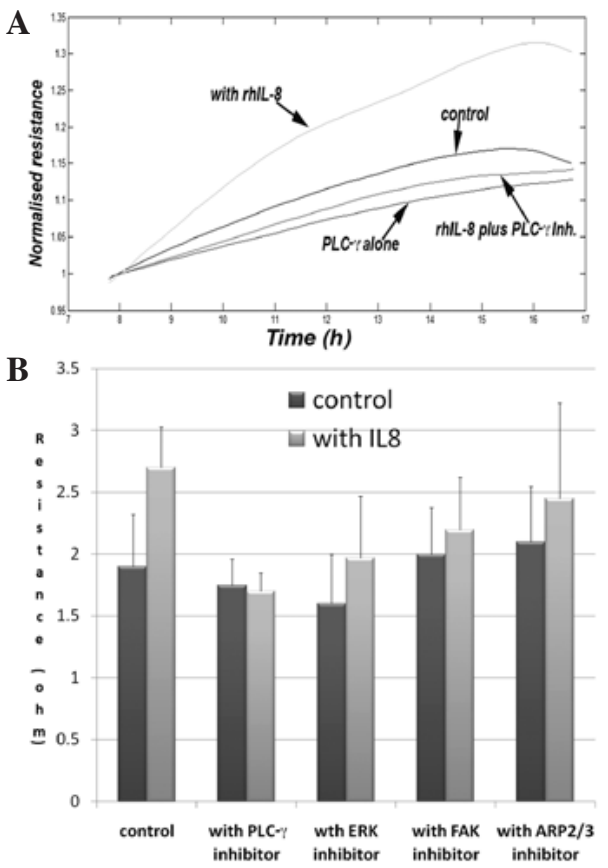

Figure 4. Potential signalling pathways involved in IL-8-mediated migration in HaCaT cells. (A) ECIS traces following electric wounding, (B) Mean changes of resistance. " $\mathrm{p}<0.05$ vs. without IL-8. $\mathrm{n}=4$. rhIL-8, recombinant human IL-8; ECIS, electric cell-substrate impedance sensing.
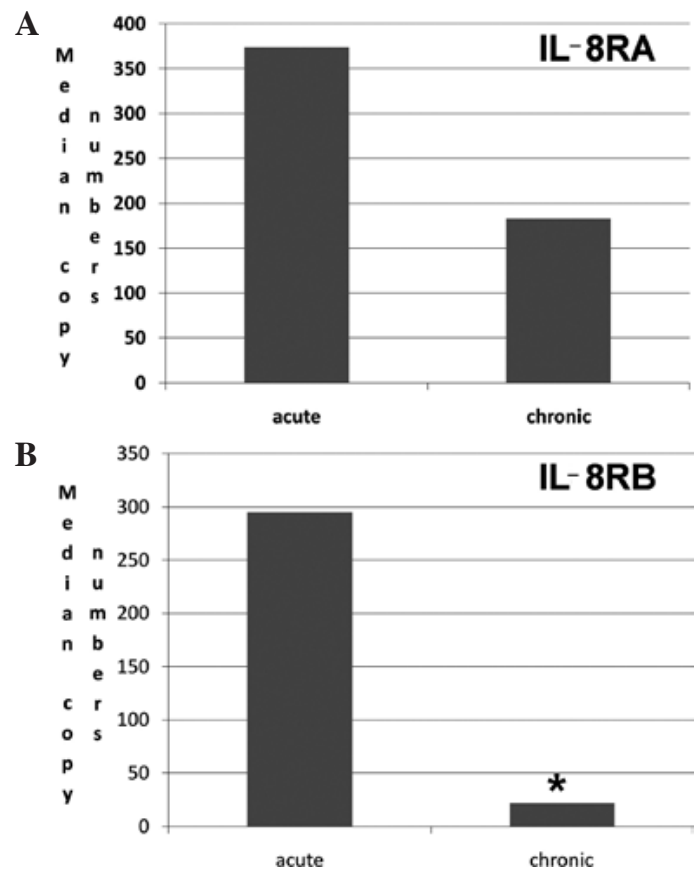

Figure 5. The levels of IL-8 receptor transcript expression in acute and chronic wounds. (A) IL-RA, (B) IL-8RB. The levels shown are the medians. " $\mathrm{p}<0.05$ vs. acute wound tissues, as shown by the Mann-Whitney U test. IL-8R, IL-8 receptor. 


\section{Discussion}

The present study reports that the status of the IL-8R, IL-8RB, is linked to clinical outcome in chronic wounds. Furthermore, this study further demonstrates that IL- 8 has a direct impact on the migration of human keratinocytes.

We show that IL- 8 has a profound effect on the migration of keratinocytes. It has previously been shown that IL-8 may act as a chemoattractant to keratinocytes, in that keratinocytes may respond to the gradient change of IL-8. In the present study, we used a novel method, ECIS, and an electrical wounding model. In this model, cells were wounded by an electric current, and the migration of cells from the surrounding areas was traced in the prescence of IL- 8 . The data presented here, therefore, clearly indicate that IL-8 also induces migration, most likely in the form of chemokinesis, of keratinocytes, another crucial form of cell migration.

CXC chemokine-induced chemotaxis involves a number of intracellular signalling pathways, classically the JAK, Src/PI3K, FAK and PLC pathways (27-30). The present study clearly demonstrates that the IL-8-induced migration of HaCaT cells (chemokinesis) critically requires the PLC- $\gamma$ pathway, in that the PLC- $\gamma$ inhibitor suppressed IL-8-induced migration and completely blocked the action of IL-8. By contrast, FAK, ARP2/3 and ERK inhibitors do not appear to signficantly affect the action of IL-8.

It has previously been shown that IL-8 is associated with wound healing in vivo (19). In an IL-8RB knockout mouse model, it was found that the rate of wound healing was reduced, thought to be the result of a reduction in monocyte recruitment to the wounding space, reduced angiogenesis and re-epithelialisation. In this study, we provide further evidence that IL-8 itself has a direct impact on the re-epithelialisation of keratinocytes.

Despite the evidence, in vitro and in vivo, that IL-8 and IL-8Rs have an impact on wound healing, it is not known how the IL-8Rs are linked to the healing of human wounds. The present study provides first-line evidence that in chronic wounds, the levels of IL-8RA and, in particular, IL-8RB are significantly lower when compared with acute wound tissues. This is noteworthy, as it indicates that a loss or reduction of IL-8Rs may be an essential part of the mechanisms of why wounds are not healing or responding to IL-8. It is highly plausible that the low levels of IL-8Rs rendered cells necessary for wound healing irresponsive or poorly responsive to IL-8, a cytokine that appears to be involved in the wound healing process. Together with in vitro data and evidence from animal studies, it is suggested that IL-8 and IL-8Rs are a useful indicator for the nature of healing of a given wound. This further suggests that IL-8 may be a means to manipulate the healing of wound tissues and may have a therapeutic value. This is likely to be manifested by the direct effect of IL-8 on keratinocytes, on endothelial cells, through the induction of angiogenesis, on immune cells for combating infection and fibroblasts during the tissue modelling process of wound healing. Of course, IL- 8 is not at all a clear wound healer without concerns. It has been shown that IL-8RB may mediate skin carcinogenesis when overexpressed or repeatedly induced (30).

In conclusion, IL-8 has a profound impact on keratinocytes by increasing the rate of cell migration. Together with the observation that IL-8Rs are markedly lower in chronic than in acute wounds, it is suggested that IL-8 and its receptors have prognostic and therapeutic value in difficult-healing wounds.

\section{Acknowledgements}

The authors wish to thank Dr Kevin Conway for his help in the collection of tissues. The authors also wish to thank the Welsh Government (A4B programme), Cardiff Partnership Fund, Cancer Research Wales and Albert Hung Foundation for their support.

\section{References}

1. Van Damme J, Decock B, Conings R, Lenaerts JP, Opdenakker G and Billiau A: The chemotactic activity for granulocytes produced by virally infected fibroblasts is identical to monocyte-derived interleukin 8. Eur J Immunol 19: 1189-1194, 1989.

2. Wolpe SD and Cerami A: Macrophage inflammatory proteins 1 and 2: members of a novel superfamily of cytokines. FASEB J 3: 2565-2573, 1989

3. Modi WS and Chen ZQ: Localization of the human CXC chemokine subfamily on the long arm of chromosome 4 using radiation hybrids. Genomics 47: 136-139, 1998.

4. Hull J, Thomson A and Kwiatkowski D: Association of respiratory syncytial virus bronchiolitis with the interleukin 8 gene region in UK families. Thorax 55: 1023-1027, 2000.

5. Smyth RL, Mobbs K, O'Hea U, Ashby D and Hart CA: The association between disease severity, cytokines and virus genotype in infants with respiratory syncytial virus (RSV) bronchiolitis. Arch Dis Child 82 (Suppl 1): A4-A5, 2000.

6. Hull J, Rowlands K, Lockhart E, Sharland M, Moore C, Hanchard N and Kwiatkowski DP: Haplotype mapping of the bronchiolitis susceptibility locus near IL8. Hum Genet 114: 272-279, 2004.

7. Srivastava M, Eidelman O, Zhang J, Paweletz C, Caohuy H, Yang Q, Jacobson KA, Heldman E, Huang W, Jozwik C, Pollard BS and Pollard HB: Digitoxin mimics gene therapy with CFTR and suppresses hypersecretion of IL-8 from cystic fibrosis lung epithelial cells. Proc Nat Acad Sci USA 101: 7693-7698, 2004.

8. Morris SW, Nelson N, Valentine MB, Shapiro DN, Look AT, Kozlosky CJ, Beckmann MP and Cerretti DP: Assignment of the genes encoding human interleukin-8 receptor types 1 and 2 and an interleukin- 8 receptor pseudogene to chromosome $2 \mathrm{q} 35$. Genomics 14: 685-691, 1992.

9. Mollereau C, Muscatelli F, Mattei MG, Vassart G and Parmentier M: The high-affinity interleukin 8 receptor gene (IL8RA) maps to the 2q33-q36 region of the human genome: cloning of a pseudogene (IL8RBP) for the low-affinity receptor. Genomics 16: 248-251, 1993.

10. Murphy PM and Tiffany HL: Cloning of complementary DNA encoding a functional human interleukin-8 receptor. Science 253: 1280-1283, 1991

11. Peral MC, Rachid MM, Gobbato NM, Huaman Martinez MA and Valdez JC: Interleukin-8 production by polymorphonuclear leukocytes from patients with chronic infected leg ulcers treated with Lactobacillus plantarum. Clin Microbiol Infect 16: 281-286, 2010.

12. Sticherling M, Bornscheuer E, Schröder JM and Christophers E: Localization of neutrophil-activating peptide-1/interleukin-8immunoreactivity in normal and psoriatic skin. J Invest Dermatol 96: 26-30, 1991

13. Cataisson C,Pearson AJ, Tsien MZ, Mascia F, Gao JL, Pastore S and Yuspa SH: CXCR2 ligands and G-CSF mediate PKCalpha-induced intraepidermal inflammation. J Clin Invest 116: 2757-2766, 2006.

14. Wettey FR, Xue L and Pettipher R: Salbutamol inhibits trypsin-mediated production of CXCL8 by keratinocytes. Cytokine 36: 29-34, 2006.

15. Trompezinski S, Bonneville M, Pernet I, Denis A, Schmitt D and Viac J: Gingko biloba extract reduces VEGF and CXCL-8/ IL-8 levels in keratinocytes with cumulative effect with epigallocatechin-3-gallate. Arch Dermatol Res 302: 183-189, 2010.

16. Sfakianakis A, Barr CE and Kreutzer DL: Localization of the chemokine interleukin- 8 and interleukin- 8 receptors in human gingiva and cultured gingival keratinocytes. J Periodontal Res 37: 154-160, 2002. 
17. Schulz BS, Michel G, Wagner S, Süss R, Beetz A, Peter RU, Kemény $\mathrm{L}$ and Ruzicka T: Increased expression of epidermal IL-8 receptor in psoriasis. Down-regulation by FK-506 in vitro. J Immunol 151: 4399-4406, 1993.

18. Michel G, Kemény L, Peter RU, Beetz A, Ried C, Arenberger P and Ruzicka T: Interleukin- 8 receptor-mediated chemotaxis of normal human epidermal cells. FEBS Lett 305: 241-243, 1992.

19. Devalaraja RM, Nanney LB, Du J, Qian Q, Yu Y, Devalaraja MN and Richmond A: Delayed wound healing in CXCR2 knockout mice. J Invest Dermatol 115: 234-244, 2000.

20. Conway K, Harrison G, Price P, Harding KG and Jiang WG: Expression of HGF, HGF receptor and the JHGF regulators in the acute and chronic wounds in the lower limb. Wound Repair Reg 15: 683-692, 2007.

21. Conway K, Harrison G, Price P, Harding KG and Jiang WG: Vascular endothelial growth inhibitor represents an important target for therapeutic angiogenesis in the lower limb. Int Wound J 4: 55-64, 2007.

22. Nazarenko IA, Bhatnagar SK and Hohman RJ: A closed tube format for amplification and detection of DNA based on energy transfer. Nucleic Acids Res 25: 2516-2521, 1997.

23. Jiang WG, Ye L, Patel G and Harding KG: Expression of WAVES, the WASP (Wiskott-Aldrich Syndrome Protein) family of Verprolin Homologous proteins, in human wound tissues determines the fate of wound healing. Wound Repair Reg 18 594-604, 2010.
24. Jiang WG, Davies G, Martin TA, Parr C, Watkins G, Mason MD and Mansel RE: Expression of membrane type-1 matrix metalloproteinase, MT1-MMP in human breast cancer and its impact on invasiveness of breast cancer cells. Int J Mol Med 17: 583-590, 2006.

25. Keese CR, Wegener J, Walker SR and Giaever I: Electrical wound-healing assay for cells in vitro. Proc Natl Acad Sci USA 101: 1554-1559, 2004.

26. Jiang WG, Martin TA, Russell-Lewis J, Ye L, Douglas-Jones A and Mansel RE: Eplin-alpha expression in human breast cancer, the impact on cellular migration and clinical outcome. Mol Cancer 7: 71, 2008.

27. Holmes WE, Lee J, Kuang WJ, Rice GC and Wood WI: Structure and functional expression of a human interleukin-8 receptor. Science 253: 1278-1280, 1991.

28. Borish LC and Steinke JW: Cytokines and chemokines. J Allergy Clin Immunol 111 (Suppl 2): S460-S475, 2003

29. Sprenger, H, Lloyd AR, Lautens LL, Bonner TI and Kelvin DJ: Structure, genomic organization, and expression of the human interleukin-8 receptor B gene. J Biol Chem 269: 11065-11072, 1994.

30. Cataisson C, Ohman R, Patel G, Pearson A, Tsien M, Jay S, Wright L, Hennings $\mathrm{H}$ and Yuspa SH: Inducible cutaneous inflammation reveals a protumorigenic role for keratinocyte CXCR2 in skin carcinogenesis. Cancer Res 69: 319-328, 2009. 